\title{
NUCLEAR MAGNETIC RESONANCE IN DILUTE ALLOYS
}

\author{
K. TOMPA \\ Central Research Institute for Physics, Budapest, Hungary
}

\begin{abstract}
The experimental results concerning the electric field gradient and the local magnetic field around substitutional impurities in copper-based dilute alloys have been summarized. Comparing the experimental data with theoretical models we have come to the conclusion that only the first-order quadrupole wipe-out number characteristic of the asymptotic charge density oscillation around the impurities can at present be properly interpreted.
\end{abstract}

\section{INTRODUCTION}

In the last ten or fifteen years the study of dilute alloys has contributed substantially to our knowledge of metals and alloys. Nevertheless. there are still many problems which require further experimental and theoretical investigations.

The study of microscopic (local) behaviour is expected to furnish new data in addition to transport, thermal and magnetic properties. The most effective tool for the study of local properties is known to be n.m.r. spectroscopy.

It is beyond the scope of this paper to review all the results achieved by n.m.r. spectroscopy. The present considerations will be restricted to the electric field gradient and the local magnetic field perturbations in the environment of the alloyant atoms (impurities) in a copper matrix. This report has been written with three objectives. First we want to summarize the latest experimental results in this field; second, the results obtained in our laboratory will be presented alongside those of other authors. Finally, the interpretation of the experimental data will be discussed in detail without. however, an attempt at the confirmation of any given theory. The alloys of copper with $3 \mathrm{~d}$ transition metals will be specifically considered. The physical properties and the anomalies observed on these alloys have been dealt with in a large number of papers. As references, three articles of a monographical character can be mentioned ${ }^{1,2,3}$.

\section{REVIEW OF EXPERIMENTAL DATA}

Before actually surveying the experimental results, it seems of interest to consider briefly the type of samples used for the experiments, their preparation and some consequences of the alloying techniques applied. 


\section{K. TOMPA}

\section{Samples}

N.m.r. measurements are usually made on powder samples; monocrystals have also been applied recently for the study of dilute alloys ${ }^{4,5}$. The samples introduced in Budapest differ from conventional powder samples. Here foils about $15 \mu \mathrm{m}$ thick are sandwiched between insulating layers ${ }^{6}$. With these sandwich-type samples the accuracy of the relative amplitude measurement of the n.m.r. spectrum is found to be around $\pm 1 \%{ }^{7}$, and so is better than that reported for powder samples. It has to be noted that the accuracy of the amplitude, i.e. intensity measurement, is of critical importance in the evaluation of the quadrupole effect.

\section{Preparation of samples}

Two non-trivial problems are worth mentioning in connection with alloying procedures. One of them is fast cooling, the other the internal oxidation.

Fast cooling is applied in order to maintain the impurity concentration in the solution. In this case the vacancies frozen along with the impurities may considerably affect the result of the measurement ${ }^{8}$.

Oxidation can occur at high temperatures when the samples happen to be under an atmosphere containing oxygen, e.g. poor vacuum with pressures from $10^{-5}$ to $10^{-4} \mathrm{mmHg}$, technical grade argon, etc. This has been observed in the early experiments on dilute copper-gallium alloys ${ }^{9}$. Depending on the length of annealing time, practically any experimental value can be obtained in a poor vacuum, e.g. even the first-order quadrupole effect wipe-out number of $n_{1}=0$ which holds for the unperturbed case (pure metal). It is known that many alloyants have a tendency to oxidation in a copper matrix ${ }^{10}$. Thus, it is not the number of the first-order quadrupole effect wipe-out number that decreases, but the number of 'active' gallium impurities that can be reduced even to zero.

\section{Experimental methods}

Various experimental setups have been used in the measurements referred to, such as the simplest continuously excited 'broad line' spectrometers, the field cycling method introduced by Redfield ${ }^{11}$ and the SEDOR (spin-echo double resonance) technique ${ }^{12}$. It is of interest to note that the simplest technique essentially contributed to the better understanding of the problems.

\section{(1) Field gradient measurements}

The literature on the quadrople effect in the n.m.r. spectrum has been reviewed $^{13}$. The quadrupole effect in dilute alloys has been discussed in theoretical papers by Friedel and Blandin ${ }^{14}$ and by Kohn and Vosko ${ }^{15}$. There are also two important experimental papers to be referred to ${ }^{16,7}$. Some results of the theoretical approaches will be discussed below.

The electric field gradient measurements can be divided into three groups, as illustrated in Figure 1.

Thus the measured values can reflect either the field gradient at the nuclei on given coordination shells or the average value of the field gradient in the range close to the impurities or that in the asymptotic range. The latter two 


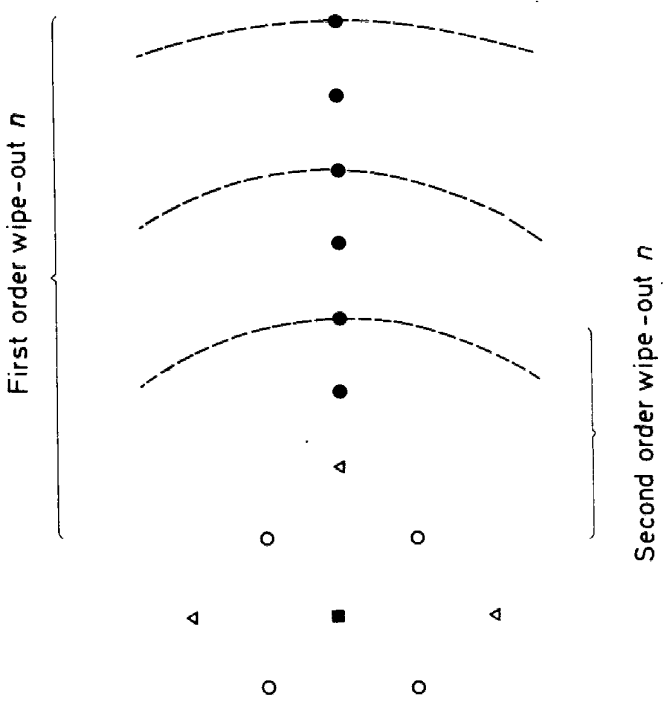

Figure 1. Separation of field gradient measurements

are characterized by the second and first order quadrupole effect wipe-out numbers $n_{2}$ and $n_{1}$ respectively.

Table 1 lists the parameter values of the field gradients measured at nuclei on the given coordination shells in dilute copper-based alloys.

Table 1. Electric field gradient (in $\AA^{-3}$ units) and asymmetry parameter for first and second nearest neighbours of impurities in copper

\begin{tabular}{lrcc}
\hline Alloy & \multicolumn{2}{c}{ First n.n. } & $\begin{array}{c}\text { Second n.n. } \\
q_{2}\end{array}$ \\
\cline { 2 - 3 } & $q_{1}$ & $\eta$ & \\
\hline$\underline{\mathrm{Cu}}-\mathrm{Zn}^{\mathrm{b}}$ & 0.75 & 0.27 & $0.04^{\mathrm{c}}$ \\
$\underline{\mathrm{Cu}}-\mathrm{Ge}^{\mathrm{a}}$ & 0.74 & 0.91 & 0.31 \\
$\underline{\mathrm{Cu}}-\mathrm{Cd}^{\mathrm{b}}$ & 0.58 & 0.04 & 0.16 \\
$\underline{\mathrm{Cu}}-\mathrm{In}^{\mathrm{a}}$ & 0.84 & 0.32 & 0.31 \\
$\underline{\mathrm{Cu}}-\mathrm{Sn}^{\mathrm{a}}$ & 0.88 & 0.64 & 0.44 \\
$\underline{\mathrm{Cu}}-\mathrm{Sb}^{\mathrm{a}}$ & 1.00 & 0.75 & 0.66 \\
$\overline{\mathrm{Cu}}-\mathrm{Ag}^{\mathrm{b}}$ & 0.22 & 0.75 & 0.18 \\
$\underline{\mathrm{Cu}}-\mathrm{Au}^{\mathrm{b}}$ & 1.01 & 0.05 & 0.31 \\
$\underline{\mathrm{Cu}}-\mathrm{Ni}^{\mathrm{d}}$ & 0.42 & 0.20 & - \\
$\underline{\mathrm{Cu}}-\mathrm{Co}^{\mathrm{e}}$ & 0.19 & 0.57 & - \\
$\underline{\mathrm{Cu}}-\mathrm{Mn}^{\mathrm{f}}$ & $\leqslant 0.05$ & - & - \\
\hline
\end{tabular}

\footnotetext{
(a) Jensen $e t$ al., ref. 5 .

(b) Schnakenberg et al., ref. 4.

(c) Redfield, ref. 11

(d) Lo et al., ref. 17.

(e) Lang et al., ref. 12.

(f) Tompa, ref. 18.
}

Table 2 shows the wipe-out numbers characteristic of the first and second order quadrupole perturbations in copper-based alloys. 


\section{K. TOMPA}

Table 2. Wipe-out numbers for the second $\left(n_{2}\right)$ and for the first $\left(n_{1}\right)$ order quadrupole effects in dilute copper-based alloys

\begin{tabular}{|c|c|c|c|c|c|c|c|}
\hline Alloy & & & $n_{1}$ & Alloy & & $n_{2}$ & $n_{1}$ \\
\hline $\mathrm{Cu}-\mathrm{Zn}$ & $18^{\mathrm{a}}$ & $17^{b}$ & $490 \pm 25^{\mathrm{e}}$ & $\mathrm{Cu}-\mathrm{Ag}$ & $25^{\mathrm{a}}$ & & $590 \pm 30^{d}$ \\
\hline $\mathrm{Cu}-\mathrm{Ga}$ & $38^{a}$ & & $900 \pm 30^{\mathrm{d}}$ & $\overline{\mathrm{Cu}} \mathrm{Au}$ & $44^{a}$ & $44^{b}$ & $880 \pm 40^{\mathrm{c}}$ \\
\hline $\mathrm{Cu}-\mathrm{Ge}$ & $63^{a}$ & & $1400 \pm 50^{\mathrm{d}}$ & $\mathrm{Cu}-\mathrm{Mn}$ & & & $1500 \pm 75^{\mathrm{e}}$ \\
\hline $\mathrm{Cu}-\mathrm{As}$ & $80^{\mathrm{a}}$ & & - & $\overline{\mathrm{Cu}}-\mathrm{Fe}$ & - & & $2100 \pm 100^{r}$ \\
\hline$\overline{\mathrm{Cu}}-\mathrm{Cd}$ & $32^{\mathrm{a}}$ & & - & $\mathrm{Cu}-\mathrm{Co}$ & - & & $2050 \pm 100^{\mathrm{g}}$ \\
\hline $\mathrm{Cu}-\mathrm{In}$ & $48^{a}$ & & - & $\mathrm{Cu}-\mathrm{Ni}$ & $19^{\mathrm{a}, \mathrm{h}}$ & $24^{\mathrm{b}} 28^{\mathrm{i}}$ & $1250 \pm 125^{\mathrm{c}}$ \\
\hline$\overline{\mathrm{Cu}}-\mathrm{Sn}$ & $67^{a}$ & & $\ldots$ & $\overline{\mathrm{Cu}}-\mathrm{Pd}$ & $38^{\mathrm{a}, \mathrm{h}}$ & $33^{b}$ & $1200 \pm 100^{\circ}$ \\
\hline $\mathrm{Cu}-\mathrm{Sb}$ & $87^{\mathrm{a}}$ & & - & $\mathrm{Cu}-\mathrm{Pt}$ & $60^{\mathrm{a}, \mathrm{h}}$ & $60^{b}$ & $1900 \pm 100^{c}$ \\
\hline
\end{tabular}

\footnotetext{
(a) Rowland $v_{0}=4 \mathrm{MHz}$, ref. 16 .

(b) Tompa $v_{0}=6 \mathrm{MHz}$, ref. 7 .

(c) Tompa, ref. 7 .

(d) Serfözö et al., ref. 19 ,

(e) Tompa, ref. 20.

(f) Tompa et al., ref. 21.

(g) Tompa, unpublished.

(h) Beal-Monod, ref. 22.

(i) Chapman et al, ref. 24 .
}

On inspection, no comparable trends can be noticed in the data of Tables 1 and 2 . The values of $n_{1}$ and $n_{2}$ in Table 2 show several similar tendencies.

\section{(2) Local magnetic field measurements}

Listing of the data obtained from measurement of the local magnetic field in the environment of impurity atoms will be restricted to the values of the magnetic field measured at nuclei on the coordination shells at a given distance from the impurity atom. The discussion will not be extended to the study started by Owen et al. ${ }^{23}$ and continued by Behringer, Van der Lugt et al., Chapman and Seymour, and Sugaware ${ }^{24}$ which concerned mainly the broadening and the shift of the n.m.r. spectrum. The coupling constant $J_{\text {sd }}$ was determined from these investigations. The anomaly of the n.m.r. spectrum at about the Kondo temperature ${ }^{25}$ will be also left out of consideration since no local magnetic field measurement has been reported on the same metal in both the non-magnetic and the magnetic state. The results of the local magnetic field measurements are listed in Table 3.

Table 3. Local magnetic fields in some copper-3d transition metal dilute alloys

\begin{tabular}{rlllc}
\hline Alloy & $\Delta K_{1} / H_{0}$ & $\Delta K_{2} / H_{0}$ & $\Delta K_{3} / H_{0}$ & Temp. K \\
\hline$\underline{\mathrm{Cu}}-\mathrm{Mn}^{\mathrm{a}}$ & $-4.87 \pm 0.2$ & & - & 300 \\
$\underline{\mathrm{Cu}}-\mathrm{Co}^{\mathrm{b}}$ & $-3.84 \pm 0.04$ & $1.91 \pm 0.02$ & -0.72 & 4.2 \\
$\underline{\mathrm{Cu}}-\mathrm{Ni}^{\mathrm{c}}$ & $-0.27 \pm 0.03$ & - & - & 4.2 \\
\hline
\end{tabular}

(a) Tompa, ref. 18. (b) Lang et al., ref. 12. (c) Lo et al., ref. 17.

\section{INTERPRETATION OF THE EXPERIMENTAL RESULTS}

\section{(1) Theories}

The theoretical relations needed for the interpretation of the experimental data are briefly referred to. According to the generally accepted definition ${ }^{26}$ 
the field gradient can be attributed to two contributions; one of them is called 'size effect' which is due to the lattice deformation caused by the impurity, and the other is called 'charge effect' caused by the conduction electron density perturbation around the impurity.

\section{Lattice deformation around impurities-size effects}

The contribution from lattice deformation appears first in terms of the continuum model of solids as the Blatt correction ${ }^{27}$

$$
\Delta Z^{\prime}=\Delta Z-\left\{3 / \gamma_{\mathrm{E}}\right\}\left\{(1 / a)(\mathrm{d} a / \mathrm{d} c) Z_{0}\right\}
$$

where $\Delta Z$ is the matrix impurity valence difference, $\gamma_{\mathrm{E}}=3(1-\sigma) /(1+\sigma)$, $\sigma$ being the Poisson ratio, $(1 / a)(\mathrm{d} a / \mathrm{d} c)$ the relative change in the lattice constant per impurity concentration, and $Z_{0}$ the charge of matrix ions.

The contribution from the lattice deformation to the electric field gradient is given by Sagalyn et $a l^{26}$ as

$$
q_{\lambda}=\lambda \frac{27}{2^{\frac{3}{2}} \pi \gamma_{\mathrm{E}}} \times \frac{1 \mathrm{~d} a}{a} \frac{\mathrm{d} c}{r^{-3}}
$$

where $r$ is the distance from the impurity and $\lambda$ is an empirical parameter. The meaning of $\lambda$ was discussed more fully in an earlier report ${ }^{28}$ and in our direct measurement it was found that $\lambda \leqslant 1.5$, which is only one tenth of the contribution to the field gradient determined by Sagalyn et al. and thus about ten per cent of the contribution from Friedel oscillation. However, this estimation does not permit any inferences to be made on the effect of lattice deformation in the neighbourhood of the impurities.

The theory of Beal-Monod and $\mathrm{Kohn}^{28}$ has to be mentioned as a comprehensive theory of lattice deformation which accounts for both the Blatt and Sagalyn effects, and also determines the validity of the Blatt correction. However, in practice one cannot use the prediction of this theory in the evaluation. The Blatt correction is the only term that we can use from the 'size' effect formulation.

Electric field gradient due to charge density oscillation around impurity

According to Kohn-Vosko's ${ }^{15}$ and Blandin-Friedel's ${ }^{14}$ asymptotic theory, the $Z$ component of the electric field gradient at the matrix nuclei around the impurity in metals of cubic symmetry can be expressed in the form

$$
q=\frac{8 \pi}{3} \alpha \Delta n|r|
$$

where $\alpha$ is the enhancement factor accounting for both the Bloch character of the conduction electrons and the Steinheimer antishielding factor ${ }^{29}$, predicted $^{15}$ for copper as 25.5 . Up to this time we have this predicted value for $\alpha$ and an estimation ${ }^{28}$ of the upper limit of $\alpha$ as $\alpha \leqslant 25$. The electric field gradient is directly related to the charge density oscillation $\Delta n|r|$, and

$$
\begin{aligned}
\Delta n|r| & =\left(A / r^{3}\right) \cos \left(2 k_{\mathrm{F}} r+\varphi\right) \\
A \sin \varphi & =\left(1 / 2 \pi^{2}\right) \sum_{l}(-1)^{l}(2 l+1) \sin ^{2} \eta_{l} \\
A \cos \varphi & =\left(1 / 2 \pi^{2}\right) \sum_{T}(-1)^{l}(2 l+1) \sin \eta_{l} \cos \eta_{l}
\end{aligned}
$$




\section{K. TOMPA}

where $k_{\mathrm{F}}$ is the Fermi wavenumber, and $\eta_{l}$ stands for the phase shifts of the scattered partial wave characterized by the quantum number $l$ at the Fermi energy. The phase shifts satisfy the Friedel sum rule

$$
Z=(2 / \pi) \sum_{l}(2 l+1) \eta_{l}
$$

which is the condition of self-consistency for the scattering potential. The methods used for the evaluation of phase shifts are summarized in ref. 7 .

For transition metal impurities the Friedel-Anderson theory ${ }^{14,}{ }^{30}$ leads to the same expression (4) as given above provided that the distance between the Fermi level and the virtual level is large and that the screening charge contains contributions from states of different symmetries. If the virtual level is close to the Fermi level, resonance scattering (i.e. the term $l=2$ ) becomes dominant, and in accordance with the Friedel sum rule, the phase shift will be $\eta_{2}=N \pi / 10$, where $N$ is the number of d electrons.

Blandin ${ }^{31}$ expressed the charge density oscillation of conduction electrons with spin $\sigma$ around the impurity having a localized magnetic moment as

$$
\Delta \rho^{\sigma}|r|=-\left(5 / 4 \pi^{2}\right) \sin \eta_{2}^{\sigma} r^{-3} \cos \left(2 k_{\mathrm{F}} r+\eta_{2}^{\sigma}\right)
$$

where $\eta_{2}^{\sigma}$ stands for the phase shift of scattered electrons with spin $\sigma$. By making use of this experession the total electron charge density oscillation is given as

$$
\Delta n|r|=\Delta \rho^{\uparrow}|r|+\Delta \rho^{\downarrow}|r|
$$

and the spin density oscillation as

$$
\Delta s|r|=\Delta \rho^{\dagger}|r|-\Delta \rho^{\downarrow}|r|
$$

On introducing the notation used by Souletie ${ }^{32}$ the description can be simplified to

$$
\begin{aligned}
\eta_{2}^{\dagger} & =(1+\xi) \eta_{2} \\
\eta_{2}^{\downarrow} & =(1-\xi) \eta_{2}
\end{aligned}
$$

According to Souletie $\xi=0$ corresponds to the 'non-magnetic' and $\xi=1$ to the 'magnetic' case. In the 'non-magnetic' case the Blandin-Souletie description corresponds to formula 4 in the case of resonance scattering. In the magnetic case $\eta_{2}^{\uparrow}=2 \eta_{2}$ and $\eta_{2}^{\frac{1}{2}}=0$ and the charge and spin density oscillations are equivalent, i.e.

$$
\Delta n|r|=\Delta s|r|=-\left(5 / 4 \pi^{2}\right) \sin 2 \eta_{2} r^{-3} \cos \left(2 k_{\mathrm{F}} r+2 \eta_{2}\right)
$$

In this simple model the charge density oscillation is brought about by the electrons polarized in spin. The amplitude of charge density oscillation in the 'non-magnetic' case is proportional to $2 \sin \eta_{2}$, in the 'magnetic' case to $\sin 2 \eta_{2}$, and the amplitude of spin density oscillation in the 'magnetic' case is proportional to $\sin 2 \eta_{2}$ and of course equal to zero when $\xi=0$.

In the case of localized moment it is easy to express in the terms of this theory the hyperfine field due to spin density oscillation ${ }^{31,33}$ as

$$
\Delta H|r|=(16 \pi / 3) \mu_{\mathrm{B}}\left\langle|\psi(0)|^{2}\right\rangle \frac{\left\langle S_{\mathrm{z}}\right\rangle}{S} \Delta s(r)
$$


where $\langle|\psi(0)|\rangle$ is, the wave function of the electron with Fermi energy at the resonant nucleus in the pure metal, $\mu_{\mathrm{B}}$ is the Bohr magneton, $\left\langle S_{z}\right\rangle$ is the thermal average, $S$ is the spin quantum number, $H_{0}$ is the external field, $k_{\mathrm{B}}$ is the Boltzmann constant, $T$ is the temperature and $\Delta s|r|$ is the spin density oscillation. $\Delta H|r|$ causes a $H_{0} / T$ dependent broadening of the n.m.r. spectrum.

$\left\langle|\psi(0)|^{2}\right\rangle$ can be determined if the Ruderman Kittel coupling constant is known ${ }^{34,35}$. This formula reproduces the RKKY formula, except for the phase factor in $\Delta s|r|$, if the coupling constant $J_{\text {eff }}$ is defined (for $\left.\xi=1\right)^{3.37}$ as

$$
J_{\text {eff }}=(2 l+1) J_{\text {sd }}=\left(10 E_{\mathrm{F}} / 3 \pi S\right) \sin 2 \eta_{2}
$$

The relations are utilized for the interpretation of the asymptoic values obtained from the measurement.

The pre-asymptotic form $^{34}$ of the charge density oscillation contains apart from the known Friedel oscillation, a term in $r^{-4}$, that is

$$
\Delta n|r|=A r^{-3} \cos \left(2 k_{\mathrm{F}} r+\varphi\right)+B r^{-4} \cos \left(2 k_{\mathrm{F}} r+\zeta\right)
$$

The definitions of $B$ and $\zeta$ are given by formula (4) in ref. 38 in the form of sums representing the phase shifts and the derivatives of the phase shifts with respect to wavenumber, taken at $k_{\mathrm{F}}$ respectively. According to the estimation of the authors $B>A$, thus the effect of $r^{-4}$ is worth considering for close neighbours.

The above relations are those which will be utilized for the interpretation of the experimental results. No numerical agreement is to be expected as the present theories and also those not referred to here neglect many possible contributions (the shape of the Fermi surface, the role of the d-electrons in copper, etc.). It will be seen that the simple asymptotic theories give an understandable explanation of the phenomena far from the impurities and that no quantitative description is available for those observed in close proximity to the impurities.

\section{(2) Interpretation of field gradient measurements}

Before reviewing the interpretation, we want to call attention once more to the problem of $\alpha$. Each evaluation accepts the $\alpha=25.5$ value, calculated by Kohn-Vosko ${ }^{15}$, as a revelation, in spite of the fact that no experimental verification is available and only an estimated upper limit is known ${ }^{28}$. Furthermore, nobody can be a priori sure about characterizing the antishielding effect in the immediate vicinity of the impurity by a scalar quantity.

\section{Asymptotic results}

In spite of the fact that in chronological order the work of Rowland ${ }^{16}$ was the first, we intend to start with the interpretation of the first-order wipe-out number for in this case, theoretically, an agreement of asymptotic theory and experimental results might be expected. The interpretations are discussed in the following groups : impurities with different valence, impurities with the same valence, $3 \mathrm{~d}$ transition metal impurities, nickel, palladium and platinum impurities.

(l) Impurity with different valence-The evaluation was performed according to formulae 3 and 4 and every phase shift system available in the 
literature was considered. The details of our evaluation and the above phase shifts are available in refs. 7 and 19. The measured wipe-out numbers were compared with those calculated according to the Langer-Vodko theory ${ }^{39}$. Within our evaluation, no other size effect than the Blatt correction was considered. The results are shown in Figure 2. The following conclusions were drawn.

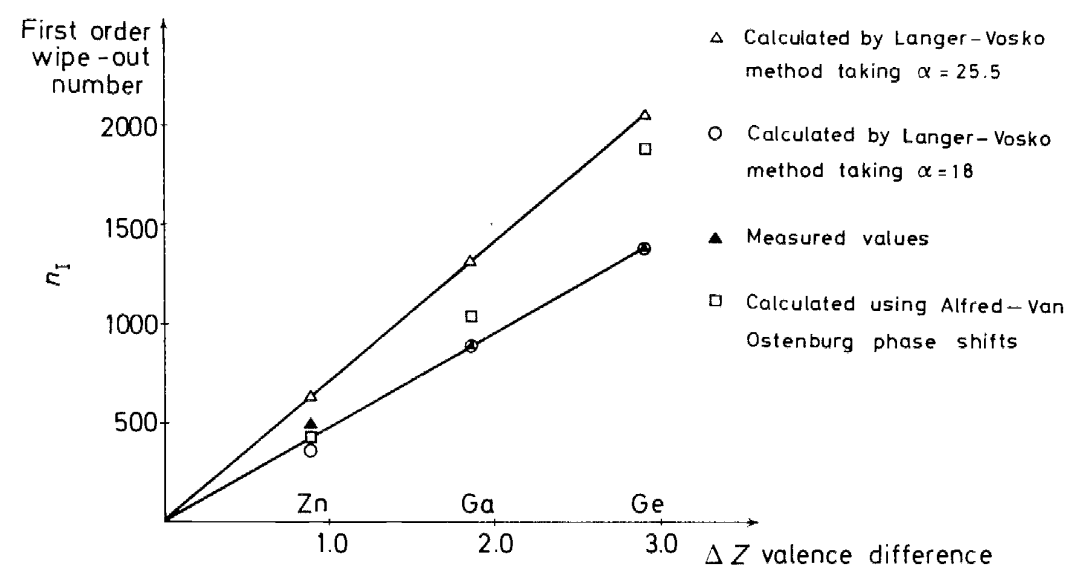

Figure 2. First-order quadrupole effect in copper-zinc, copper-gallium and copper-germanium dilute alloys

The experimental results are described by Langer-Vosko theory only in the case we are calculating with the $\alpha=18$ enhancement factor instead of $\alpha=25.5$.

Regarding the phase shifts available in the literature, the best agreement is obtained by using those of Alfred-Van Ostenburg.

The first-order wipe-out numbers calculated by the Kohn-Vosko phase shifts are some 60 per cent larger than the measured values.

Considering further measured quantities (impurity resistivity, Knight shift measured on the melt) we came to the conclusion that the three measured quantities are not described properly by any of the known phase shift systems.

We tried ${ }^{19}$ to get a graphical solution based on the Faber-Ziman diagram of the relation concerning the three physical quantities. The 'empirical' phase shifts and the values of the $n_{1}$, impurity resistivity and of the Knight shift, calculated by them, are summarized in Table 4 . The results, shown in Table 4, seem to prove the existence of a phase-shift system by which the experimental results could be described. The elaboration of a 'theoretical' phase-shift system, by which the quantities measured experimentally could be more exactly described, would be an important step.

(2) The situation is even worse with silver and gold as impurities, if evaluation is performed similarly to that carried out for zinc, gallium and germanium impurities ${ }^{19,7}$. In this approach the Kohn-Vosko phase shift, giving the best agreement for the silver impurity, describes well the Knight shift measured on the melt, but gives about 60 per cent of the measured first-order quadrupole wipe-out number. The $n_{1}$ is described well for the gold impurity, but the 
NMR IN DILUTE ALLOYS

Table 4. Empirical phase shifts, first-order wipe-out number, impurity resistivity and Knight shift (in melts) calculated on the basis of Faber-Ziman diagrams

\begin{tabular}{|c|c|c|c|c|c|}
\hline \multirow[t]{2}{*}{ Alloys } & \multicolumn{3}{|c|}{ Phase shifts } & \multicolumn{2}{|c|}{$n_{1}$} \\
\hline & $\eta_{0}$ & $\eta_{1}$ & $\eta_{2}$ & meas. & calc. \\
\hline $\mathrm{Cu}-\mathrm{Zn}$ & 0.22 & 0.24 & 0.05 & 490 & 400 \\
\hline$\underline{\mathrm{Cu}}-\mathrm{Ga}$ & 0.52 & 0.57 & 0.15 & 900 & 820 \\
\hline \multirow[t]{3}{*}{$\underline{\mathrm{Cu}}-\mathrm{Ge}$} & 0.71 & 0.90 & 0.15 & 1400 & 1800 \\
\hline & \multicolumn{2}{|c|}{$\Delta \rho[\mu \mathrm{ohm} \mathrm{cm} / \mathrm{at} . \%]$} & \multicolumn{2}{|c|}{$-(1 / K)(\partial K / \partial c)$} & \\
\hline & meas. & calc. & meas. & calc. & \\
\hline $\mathrm{Cu}-\mathrm{Zn}$ & 0.33 & 0.33 & 0.19 & 0.25 & \\
\hline$\overline{\mathrm{Cu}}-\mathrm{Ga}$ & 1.42 & 1.50 & 0.60 & 0.49 & \\
\hline$\overline{\mathrm{Cu}}-\mathrm{Ge}$ & 3.75 & 3.905 & 1.07 & 0.90 & \\
\hline
\end{tabular}

Knight shift is described with a wrong sign. We suppose that neglecting the size effect results in the greatest error. The interpretation of the experimental data is not at all adequate.

(3) We might start the interpretation of the first order wipe-out numbers $^{20,21,7}$ measures on copper-based dilute alloys containing $3 \mathrm{~d}$ transition metal impurities, according to the relations 3,6 and 9. Presuming a dominant resonance scattering, we do not think the size effect will have an important part. In Figure 3 the values of charge density oscillation amplitudes for a copper-3d transition metal row and concerning the 'non-magnetic' and 'magnetic' limits are shown according to the simple model outlined above. The charge density oscillation amplitude scale and the measured wipe-out

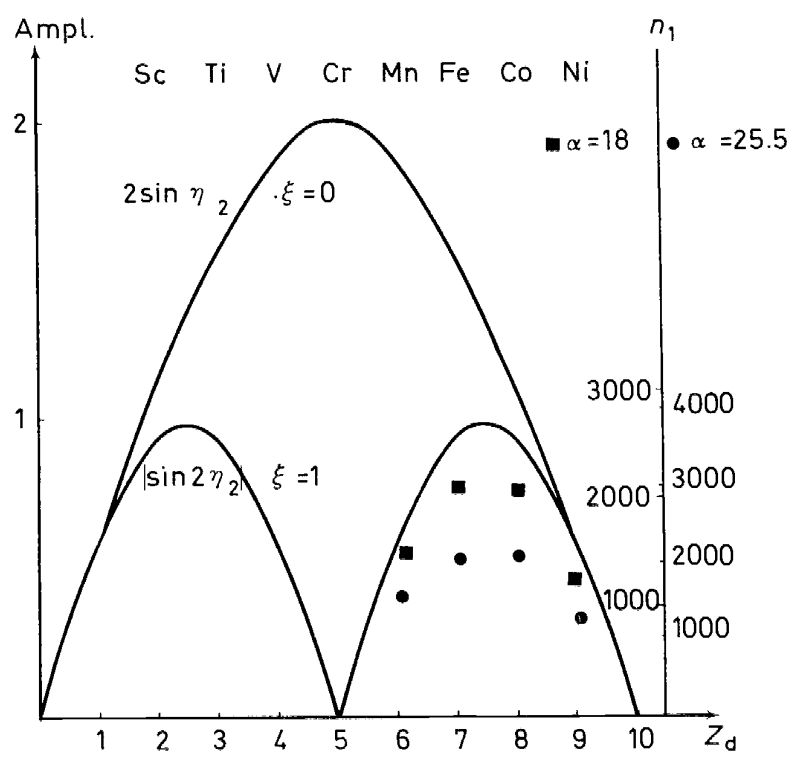

Figure 3. Charge and spin density oscillation amplitude and first-order quadrupole wipe-out numbers in copper-3d transition metal dilute alloys 


\section{K. TOMPA}

numbers were presented assuming 'resonance only' scattering. (Considering non-resonant scattering in addition, the two curves become asymmetric for the $Z_{\mathrm{d}}=5$ line; and on the right side of the Figure 3 the amplitude of $\eta_{2}$ and $n_{1}$ are also decreasing. Among the impurities manganese and iron are considered to be in the magnetic state and cobalt and nickel to be nonmagnetic. No numerical agreement is expected, but we are reassured to find the most characteristic features of the measurement data represented by the model. As for cases of impurities with different valence, it was observed that explicitly better agreements were obtained with a smaller $\alpha$ (in Figure 3 $\alpha=18$ is shown).

The model is of a very interesting consequence : in the $\xi=1$ magnetic case from the $n_{1} \sim \Delta n|r|=\Delta s|r|$ (formula 10) measurement, the $\Delta s|r|$ amplitude and the $J_{\text {eff }}$ could be determined for known $S^{37}$. After the calculations were performed - presuming only resonant scattering - the following results were obtained for copper-manganese, $J_{\text {eff }}=1.6 \mathrm{eV}(\alpha=25.5), J_{\text {eff }}=2.3 \mathrm{eV}$ $(\alpha=18)$ and presuming that $\eta_{0}$ and $\eta_{1}$ were the same as those for nickel impurities $^{7} J_{\text {eff }}=2.1 \mathrm{eV}$ was obtained. For copper-iron and in all the three cases $3.2 \mathrm{eV}, 4.5 \mathrm{eV}$ and $3.8 \mathrm{eV}$ were obtained.

(4) There is nothing more to say about the copper-platinum, copperpalladium and copper-nickel rows apart from the discussion in ref. 7. The empirical phase-shift system $\left(\eta_{0}, \eta_{1}, \eta_{2}\right)$ described there is acceptable from the physical viewpoint; however, theoretically determined phase shifts are unknown.

\section{Pre-asymptotic results}

Interpreting the wipe-out numbers characteristic of the second-order quadrupole effect, we refer to the studies of Rowland ${ }^{16}$ and of Sagalyn et al. ${ }^{26}$. We want to make some remarks on these studies and the problem itself. The $n_{1}$ wipe-out numbers are significantly overestimated in the asymptotic range by the Kohn-Vosko phase shifts used by them. Sagalyn et al. ${ }^{26}$ overestimated by a factor of ten the $\lambda$ parameter of the size effect ${ }^{28}$. Serfözö ${ }^{40}$ obtained good agreement on copper-zinc, copper-gallium and coppergermanium alloys using the Alfred-Van Ostenburg pre-asymptotic formula (13). At the same time the lattice deformation theory of $\mathrm{Nagai}^{41}$ gave the $n_{2}=33$ wipe-out number ${ }^{19}$ against the measured $n_{2}=24$. According to our opinion, the problem cannot be regarded as solved.

The interpretation of the field gradient measured at the place of the first and second neighbours was done by Jensen et al. ${ }^{5}$ and by Schnakenberg et al. $^{4}$. These field gradients calculated by the Alfred-Van Ostenburg ${ }^{38}$ self-consistent shielding potential and the Hurd-Gordon phase shifts and phase shift derivatives ${ }^{42}$, are not sufficient, according to the unanimous opinion of the authors. (In the model the field gradient has cylindrical symmetry and so $\eta=0$.) Similarly, the field gradient is not sufficient either, when calculated by Kohn-Vosko phase shifts and the asymptotic formula (5). We are of the same opinion as those authors.

Considering the field gradient measured in the neighbourhood of the $3 \mathrm{~d}$ transition metal impurities, no attempts were made to provide theoretical interpretations. Their low values, as compared to those of the other alloys, are remarkable anyway. 


\section{(3) The interpretation of the local magnetic-field measurements}

The results of Table 3 cannot be interpreted by the relation 11 or by the RKKY formula. At the place of the first neighbours a positive shift is calculated in all the three cases ${ }^{12,17,18}$ against the measured negative shift; however, this 'disagreement' is not at all surprising ${ }^{43}$. Lo et al. ${ }^{17}$ analysed the results and came to the conclusion that in copper-nickel and in coppercobalt the quotient of the relative shift $\left(\Delta K_{1} / H_{0}\right)$ and $J_{\text {sd }} x$ (s-d coupling constant, impurity susceptibility) is the same, as the consequence of the simplest

Table 5. Interpretation of local magnetic fields in some copper $-3 \mathrm{~d}$ transition metal dilute alloys

\begin{tabular}{|c|c|c|c|}
\hline Alloy & $\underline{\mathrm{Cu}}-\mathrm{Mn}^{\mathrm{c}}$ & $\underline{\mathrm{Cu}}-\mathrm{Co}^{\mathrm{d}}$ & $\underline{\mathrm{Cu}}-\mathrm{Ni}^{\mathrm{d}}$ \\
\hline (a) $\left(\Delta K_{1} / H_{0}\right)$ & $-4.87 \times 10^{-3}$ & $-3.84 \times 10^{-3}$ & $-0.24 \times 10^{-3}$ \\
\hline (b) $x(\mathrm{emu} / \mathrm{at})$ & $16 \times 10^{-27}$ & $4.0 \times 10^{-27}$ & $0.24 \times 10^{-27}$ \\
\hline \multicolumn{4}{|l|}{$(|a / b|) / 10^{24}$} \\
\hline$\left[(\mathrm{emu} / \mathrm{at})^{-1}\right]$ & 0.30 & 0.96 & 1.0 \\
\hline \multirow[t]{2}{*}{$J_{\mathrm{sd}}(\mathrm{eV})$} & 0.32 & 1.19 & 1.35 \\
\hline & $\begin{array}{l}0.42 \\
0.94\end{array}$ & 1.1 & \\
\hline$\left|a / J_{\mathrm{sd} . b}\right|$ & $\begin{array}{l}0.74 \\
0.71\end{array}$ & 0.80 & 0.74 \\
\hline
\end{tabular}

(c) Room temp. values, and $J_{\mathrm{sd}}=J_{\mathrm{eff}} /(2 \mathrm{I}+1)$ from our mentioned results $J_{\text {eff }}=1.6 \mathrm{eV}$ and $J_{\mathrm{eff}}=1.1 \mathrm{eV}$ respectively. (d) Lo et al., ref. 17 .

relation 11 . Their results and a similar analysis for copper-manganese are presented in Table 5. However, we have to admit that numerical agreement depends very much on the value of $J_{\text {sd }}$ and in this field, authors have an opportunity to choose from a wide variety.

\section{CONCLUSIONS}

We do not intend to repeat our observations about the interpretation, we only want to emphasize some of the circumstances. It is obvious that the key to development is improvement of the theories of the problem. The research worker dealing with experiments does not like to speak about an 'agreement' with theory, when the theoretical model contains several approximations, the effect of which cannot be estimated. We have to mention the unanimous acceptance of the $\alpha=25.5$ value, in spite of the fact that Kohn and Vosko accepted as correct the value of $\alpha$ within a factor of two and in spite of the fact that its experimental value is unknown. We intended to refer to this fact repeatedly in this paper, namely, that a smaller $\alpha$ offers better results. We wanted to show that the solution of the $\alpha$ problem is of the same importance as the determination of the relation concerning the appropriate charge density or the lattice deformation. Finally we want to establish, that nowwith the exception of copper-silver and copper-gold dilute alloys-the experimental results for the asymptotic range have the best interpretation.

\section{ACKNOWLEDGEMENT}

Thanks are due to Professor L. Pál and Dr B. Vasvári for supporting this work, and to my colleague Dr Cs. Hargitai for valuable discussions. 


\section{K. TOMPA}

\section{REFERENCES}

1 J. Kondo, Solid State Physics, 23, 184 (1969).

2 A. J. Heeger, Solid State Physics, 23, 283 (1969).

3 A. Narath, Crit. Rev. Solid State Sci. 3, 1 (1972).

${ }^{4}$ R. T. Schumacher and G. Schnakenberg, Solid State Commun. 7, 1735 (1969).

G. Schnakenberg and R. T. Schumacher, Phys, Rev. B, 7, 2292 (1973).

5 B. L. Jensen, R. Nevald and D. L. Williams, J. Phys. F, 2, 169 (1972).

${ }^{6}$ K. Tompa and F. Tóth, Physica Status Solidi, 3, 2051 (1963).

7 K. Tompa, J. Phys. Chem. Solids, 33, 163 (1972).

${ }^{8}$ M. A. Adawi, KFKI preprint, to be published.

9 A. Lovas, G. Serfözö, J. Tóth and K. Tompa, KFKI-preprint, to be published,

10 D. Howling, Phys. Rev. 155, 642 (1967).

11 A. Redfield, Phys. Rev. 130, 589 (1963).

12 D. V. Lang, J. B. Boyce, D. C. Lo and C. P. Slichter, Phys. Rev. Letters, 29, 776 (1972)

13 A. Abragam, The Principles of Nuclear Magnetism, Clarendon Press: Oxford (1961).

14 J. Friedel, Phil. Mag. 43, 153 (1952).

A. Blandin and J. Friedel, J. Phys. Radium, 21, 689 (1960).

15 W. Kohn and S. H. Vosko, Phys. Rev. 119, $912(1960)$.

16 T. J. Rowland, Phys. Rev. 119, 900 (1960).

T. J. Rowland and N. Shiotani, American Institution of Mechanical Engineers Conference (1965).

17 D. C. Lo, D. V. Lang, J. B. Boyce and C. P. Slichter, Phys. Rev. B, 8, 973 (1973).

${ }^{18} \mathrm{~K}$. Tompa, KFKI preprint, to be published in Phys. Status Solidi.

19 G. Serfözö, K. Tompa and A. Lovas, KFKI preprint, to te published.

20 K. Tompa, Proc. Low Temp. Phys. LT-12, Kyoto (1970). KFKI preprint 70-31 SSP.

21 K. Tompa, A. Lovas and L. Zámbó, Phys. Status Solidi (b), 54, K17 (1972).

22 M. Beal-Monod, Phys. Rev. 164, 360 (1967).

23 J. Owen, M. E. Browne, W. D. Knight and C. Kittel, Phys. Rev. 102, 1501 (1956).

J. Owen, M. E. Browne, V. Arp and A. F. Kip, J. Phys. Chem. Solids, 2, 85 (1957).

${ }^{24}$ R. E. Behringer, J. Phys. Chem. Solids, 2, 209 (1957).

W. Van der Lugt, N. J. Poulis and W. P. A. Hass, Physica, 25, 97 (1959).

A. C. Chapman and E. W. F. Seymour, Proc. Phys. Soc. 72, 747 (1959).

T. Sugawara, J. Phys. Soc. Japan, 14, 643 (1959).

25 D. C. Golibersuch and A. J. Heeger, Phys. Rev. 182, 584 (1969).

26 P. L. Sagalyn, A. Paskin and R. Harrison, Phys. Rev. 124, 428 (1961).

${ }^{27}$ F. J. Blatt, Phys. Rev. 108, 285 (1967).

${ }^{28}$ K. Tompa, F, Tóth and E. Nagy, Phys. Status Solidi, 41, 413 (1970).

29 R. M. Steinheimer and M. M. Foley, Phys. Rev. 102, 731 (1956).

30 J. M. Brettell and A. J. Heeger, Phys. Rev. 153, 319 (1967).

31 A. Blandin, $J$. Appl. Phys. 39, 1285 (1964).

32 J. Souletie, J. Low Temp. Phys. 7, 141 (1972).

33 A. Blandin, Varenna Lecture Notes (1966).

${ }^{34}$ K. Tompa, Phys. Status Solidi, 18, 391 (1966).

35 E. R. Andrew, Magnetic Resonance and Related Phenomena, p 18, ed. V. Hovi. North Holland Elsevier: Amsterdam (1973).

36 Y. Yoshida, Phys. Rev. 106, 893 (1957).

37 K. Tompa, KFKI preprint-73-31.

38 L. C. R. Alfred and D. O. Van Ostenburg, Phys. Letters, 26A, 27 (1969).

39 J. S. Langer and S. H. Vosko, J. Phys. Chem. Solids, 12, 196 (1969).

40 G. Serfözö, KFKI preprint, to be published.

41 O. Nagai, J. Phys. Soc. Japan, 20, 509 (1965).

42 C. M. Hurd and E. M. Gordon, J. Phys. Chem. Solids, 29, 2205 (1968).

43 D. J. W. Geldart, Phys. Letters A, 38, 25 (1972). 